\title{
Coronary Artery Involvement Following Kawasaki Disease: A Case Report of a 5 Month Old African Infant
}

\author{
Tumwebaze Hilda*, Ndagiire Emma, Namuyonga Judith, Twalib Aliku, Lwabi Peter and Lubega \\ Sulaiman
}

Uganda Heart Institute, Mulago Complex, Kampala, Uganda

*Corresponding author: Tumwebaze Hilda, Uganda Heart Institute, Mulago Complex, Uganda

\begin{abstract}
Background: Kawasaki disease (KD) is an acute systemic vasculitis which progresses to cause coronary artery abnormalities as a complication. Echocardiographic and cardiac angiographic data indicate that $20-40 \%$ of untreated KD patients develop coronary artery abnormalities. However, majority of the lesions regress over time. Timely treatment with high dose intravenous immunoglobulins (IVIG) and high dose of aspirin has been found to reduce the incidence of developing coronary artery aneurysms to $2 \%-3 \%$. Data on $\mathrm{KD}$ in the African region is unavailable. We present a case of a 5 month old infant that presented at the Uganda Heart Institute with KD associated with coronary artery involvement and is currently undergoing follow up.
\end{abstract}

Case presentation: A 5-months-old female infant presented to the pediatric emergency unit with a persistent high grade fever for 7 days with refusal to breast feed, diarrhea and irritability, a generalized maculopapular rash that had started 2 days after onset of fever. Her vaccination status was up to date. She was evaluated and initially managed for gastroenteritis and septicemia with no improvement. A pediatric cardiology review was done at day 22 of illness due to symptoms of difficulty in breathing and palpitations. Significant physical findings were of a very sick infant, febrile with generalized maculopapular rash, peeling of extremities and perineum, hyperemia of the conjunctiva bilaterally and the pharynx, no cervical lymphadenopathy. She had tachypnea, tachycardia with a gallop rhythm but no murmurs, tender hepatomegally, irritability but conscious with no signs of meningeal irritation.

A transthoracic echocardiography done significantly showed dilated coronary arteries, moderate pericardial effusion with preserved ejection fraction. Laboratory findings were of leucocytosis predominantly neutrophilia, thrombocytosis, anemia, raised C-reactive protein levels, hypoalbuminemia and raised liver function tests. Blood cultures and blood smear for malaria were unremarkable.
A diagnosis of $\mathrm{KD}$ with coronary artery involvement was made by the pediatric cardiologist.

Treatment for KD was started immediately with high dose intravenous immunoglobulins (IVIG) as a single dose and high dose aspirin with remarkable improvement as the fever subsided with in 24 hours of treatment. Follow up serial transthoracic echocardiography findings showed regression of coronary artery dilatation over time and currently at 3 years of age the coronary arteries are of normal diameter for her body surface area.

\section{Introduction}

Kawasaki disease (KD) is an acute systemic vasculitis which progresses to cause coronary artery abnormalities in $20-40 \%$ of untreated patients [1]. KD has a universal distribution and has been found in children of different ethnicities worldwide [2]. Its prevalence is higher in Asian countries such as Japan, where the annual incidence is 264 per 100,000 children [3] and low in non Asian countries [4,5]. Children aged 6 months to 5 years are most susceptible, with peak incidence in children aged 9-11 months [6].

The aetiology of KD is largely unknown [7]. However it is currently believed that there is a genetic predisposition to the development of KD and the interaction of an unknown infective cause $[8,9]$. Diagnosis of $\mathrm{KD}$ relies on clinical suspicion with no gold standard diagnostic test. The criteria require the presence of fever for at least 5 days along with four of five other clinical features [2] (see Table 1). Because coronary aneurysms in young children are as typical for KD,

Citation: Hilda T, Emma N, Judith N, Aliku T, Peter L, et al. (2019) Coronary Artery Involvement Following Kawasaki Disease: A Case Report of a 5 Month Old African Infant. Int Arch Cardiovasc Dis 3:025. doi.org/10.23937/2643-3966/1710025

Accepted: November 05, 2019; Published: November 07, 2019

Copyright: (C) 2019 Hilda T, et al. This is an open-access article distributed under the terms of the Creative Commons Attribution License, which permits unrestricted use, distribution, and reproduction in any medium, provided the original author and source are credited. 
Table 1: Diagnostic criteria for Kawasaki disease. Four of these five plus a fever of more than 5 days confirms the diagnosis of $\mathrm{KD}$ [2].

\begin{tabular}{|l|l|}
\hline Criterion & Description \\
\hline Conjunctivitis & Bilateral, painless, non exudative \\
\hline Lymphadenopathy & Cervical, usually more than $1.5 \mathrm{~cm}$, more commonly unilateral \\
\hline Skin rash & Commonly maculopapular \\
\hline Mucosal changes & $\begin{array}{l}\text { Red, cracked lips, glossitis with hyperplastic fungiform, papillae seen as strawberry tongue, diffuse } \\
\text { erythema of the oral mucosa or oropharynx }\end{array}$ \\
\hline Changes of extremities & $\begin{array}{l}\text { Initial stage: Erythema and oedema of palms and soles. } \\
\text { Convalescent stage: Peeling of skin from fingertips }\end{array}$ \\
\hline
\end{tabular}

Table 2: Some of the laboratory results during the course of illness.

\begin{tabular}{|c|c|c|c|c|}
\hline Days of admission & WBC(×103/ul) & Neutrophil(×103/ul) & $\mathrm{HB}(\mathrm{g} / \mathrm{dl})$ & $\operatorname{PLT}\left(\times 10^{9} / \mathrm{ul}\right)$ \\
\hline 1 & 26.44 & 23.35 & 10.0 & 484 \\
\hline 9 & 23.79 & 14.23 & 6.7 & 523 \\
\hline 17 & 19.34 & 10.95 & 6.1 & 1113 \\
\hline 24 ( 2 days after initiating treatment) & 6.27 & 1.38 & 14.4 & 967 \\
\hline
\end{tabular}

WBC: White blood cell count; HB: Hemoglobin; PLT: Platelets.

their presence as shown by echocardiography or angiography is accepted as one of the diagnostic criteria with four or less of the other criteria present to establish the diagnosis [10]. An algorithm by the American Heart Association in 2004 suggested additional use of supportive laboratory testing that included markers of inflammation, presence of anemia, leukocytosis, thrombocytosis, hypoalbuminemia, elevated liver enzymes, sterile pyuria [11].

Coronary artery involvement remains the most important complication after KD and it is assessed serially with echocardiography primarily by quantifying internal coronary artery diameters [12] and using the Z-score based on the body surface area where by a Z-score $>$ 2.5 indicates coronary artery dilatation [13]. The risk of coronary artery abnormalities can be prevented by timely administration of intravenous immunoglobulin (IVIG) and the medium to long term prognosis is excellent $[7,9]$.

The American Heart Association recommends that patients be treated with a single infusion of IVIG over twelve hours at a dosage of $2 \mathrm{~g} / \mathrm{kg}$ within ten days of fever onset, along with an anti-inflammatory $100 \mathrm{mg} / \mathrm{kg} /$ day dose of aspirin (acetylsalicylic acid) spread out over 4 doses until the child is fever free [11]. Administration of high dose intravenous IVIG has been found to reduce the incidence of coronary aneurysms significantly to $2 \%-3 \%$ if given within the first 10 days [14]. Resolution within 1-2 years after onset has been observed in approximately $50 \%-60 \%$ of coronary aneurysms, however giant aneurysms (> $8 \mathrm{~mm}$ in diameter) tend to persist [15]. Although, there is plenty of data on KD in the rest of the world, there is no available data in the African region. This case report reminds clinicians to have high index of suspicion for KD in Uganda and the rest of the African region. We describe a case of $K D$ in a five- month-old infant with coronary artery involvement in Uganda in Africa.

\section{Case Report}

A 5-months-old female infant presented to the pediatric emergency unit with persistent high grade fever for 7 days that was not associated with convulsions, was refusing to breast feed, had diarrhea but no vomiting and was irritable. She had a generalized maculopapular rash that had started 2 days following onset of the fever, had no cough. No history of contact with a person with similar symptoms was elicited and her vaccination was up to date. On admission significant laboratory findings were of leucocytosis of $26.6 \times 10^{9} / \mathrm{mm}^{3}$ predominantly neurophilia of $23 \times 10^{9} / \mathrm{mm}^{3}$, anemia with hemoglobin of $10 \mathrm{~g} /$ $\mathrm{dl}$, thrombocytosis of $484 \times 10^{9} / \mathrm{mm}^{3}$, hypoalbuminea of $24.8 \mathrm{~g} / \mathrm{l}$. Blood culture and urinalysis were normal. She was then admitted on pediatric ward managed for gastroenteritis and septicemia with antibiotics and antipyretics with no improvement. Over the days following admission her condition worsened and she developed difficulty in breathing and palpitations. A pediatric cardiology review done on day 22 revealed a febrile, infant axillary temperature $39.2{ }^{\circ} \mathrm{C}$, generalized maculopapular rash with peeling of extremities (mainly hands and feet), hyperemia of the conjunctiva bilaterally and the oral pharynx, excoriation in the genital area, had no cervical lymphadenopathy. She was tachypneic with a respiratory rate of 66 breath per minute, oxygen saturation at room air was $98 \%$, had subcostal recession with normal air entry into the lungs. She had a tachycardia of 178 beats per minute with a gallop. Heart sounds were of normal intensity and had no murmurs, blood pressure was 96/54 $\mathrm{mmHg}$. There was a tender hepatomegaly measuring $4 \mathrm{~cm}$ below the costal margin. The infant was very 
irritable but was fully conscious with no signs of meningeal irritation.

A repeat of the laboratory tests revealed persistent leucocytosis with neutrophilia, thrombocytosis, worsening anemia that had drooped up to $6.1 \mathrm{~g} /$ $\mathrm{dl}$ and C-reactive protein of $64.5 \mathrm{mg} / \mathrm{l}$ (see Table 2 for laboratory results done over the time of illness). Echocardiography findings showed dilated coronary arteries (proximal left main coronary artery was 5.4 $\mathrm{mm}$ in diameter and right coronary artery was 5.7 $\mathrm{mm}$ in diameter) and a moderate pericardial effusion.

A diagnosis of KD with coronary artery involvement was made on the basis of the clinical presentation that included fever for over 5 days, generalised maculopapular rash, peeling of the hands and feet, hyperemia of the conjunctiva bilaterally (see Table 1) and dilated coronary arteries on echocardiography. Supportive laboratory findings included leukocytosis predominantly neutrophils, anemia, thrombocytosis, raised CRP and hypoalbuminemia.

Treatment was started on day 22 with high dose infusion IVIG at $2 \mathrm{~g} / \mathrm{kg}$ (single dose) and high dose aspirin at $100 \mathrm{mg} / \mathrm{kg}$ in four divided doses for 4 days and was stepped down to $3 \mathrm{mg} / \mathrm{kg}$ for 6 weeks. The infant markedly improved with fever subsiding within 24 hours of commencing treatment. Serial follow up echocardiograms showed resolution of the dilated coronary arteries over time until child was 3-years old when the coronary arteries were of normal diameter for her body surface area (see Table 3 and Figure).

\section{Discussion}

Coronary artery lesions represent serious complications of Kawasaki disease. Commonly used definitions of coronary artery involvement have relied on the Japanese Ministry of Health criteria, which dichotomously define abnormalities as a maximum absolute internal diameter $>3 \mathrm{~mm}$ in children $<5$ years of age or $>4 \mathrm{~mm}$ in children 5 years and older, or a segment 1.5 times greater than an adjacent segment, or the presence of luminal irregularity $[12,16]$. However recent studies have used regression equations based on measurements from non febrile normal children to calculate $z$ scores based on body surface area and these allow for continuous assessment of the time course of coronary artery involvement and $z$ score of $>2.5$ is considered for dilated coronary arteries $[13,17]$. The infant we have presented at diagnosis had dilated coronary arteries (left main coronary artery diameter was $5.4 \mathrm{~mm}$ and right coronary artery diameter was $5.7 \mathrm{~mm}$ ) as per the Japanese ministry of health criteria and de Zorzi, et al. where by the $z$ scores of both coronary arteries were $>2.5$ for her body surface area (z scores for both coronary arteries was 6.6).

Several risk factors associated with coronary artery involvement have been identified, such as younger patient age and male gender, laboratory abnormalities (such as higher white cell or neutrophil count, higher C-reactive protein, higher erythrocyte sedimentation rate, lower serum albumin, and lower hemoglobin or hematocrit, treatment delay, and persistent or prolonged fever $[18,19]$. The identified risk factors for coronary artery involvement in this infant included younger patient age of 5 months, prolonged and persistent fever that had lasted 3 weeks, treatment delay that was started 22 days after onset of disease, laboratory abnormalities (such as leucocytosis with a neutrophilia, raised CRP, lower serum albumin of $24 \mathrm{~g} / \mathrm{l}$, and lower hemoglobin that kept on dropping over course of disease. However, this infant's delay in the diagnosis and thereby increasing the risk of coronary artery dilatation could be attributed to the rare occurrence of KD in the African region. Therefore this case calls for clinicians in this region to have a high index of suspicion for early diagnosis and treatment of KD to reduce the risk of developing coronary artery complications.

Despite delayed diagnosis the infant was immediately started on infusion of high dose IVIG at $2 \mathrm{~g} / \mathrm{kg}$ for 12 hours (single dose) and high dose of aspirin at $100 \mathrm{mg} / \mathrm{kg}$ in four divided dosed for 4 days and was stepped down to $3 \mathrm{mg} / \mathrm{kg}$ for 6 weeks. Treatment is recommended to start with 10 days of onset of disease

Table 3: Coronary artery diameters and $\mathrm{Z}$ score at different times during follow up of the patient.

\begin{tabular}{|l|l|l|l|}
\hline Timing & Coronary artery & Diameter $(\mathbf{m m})$ & Z score \\
\hline At first diagnosis & Left main & 5.4 & 6.6 \\
& Right & 5.7 & 6.6 \\
\hline 6 weeks later & Left main & 6.0 & 6.7 \\
\hline 6 month after diagnosis & Right & 6.6 & 6.9 \\
\hline 1 year after diagnosis & Left main & 4.0 & 2.7 \\
\hline At 3 years of age & Right & 3.0 & 3.0 \\
\hline & Left main & 3.3 & 3.2 \\
\hline & Right & 3.0 & 2.1 \\
\hline
\end{tabular}



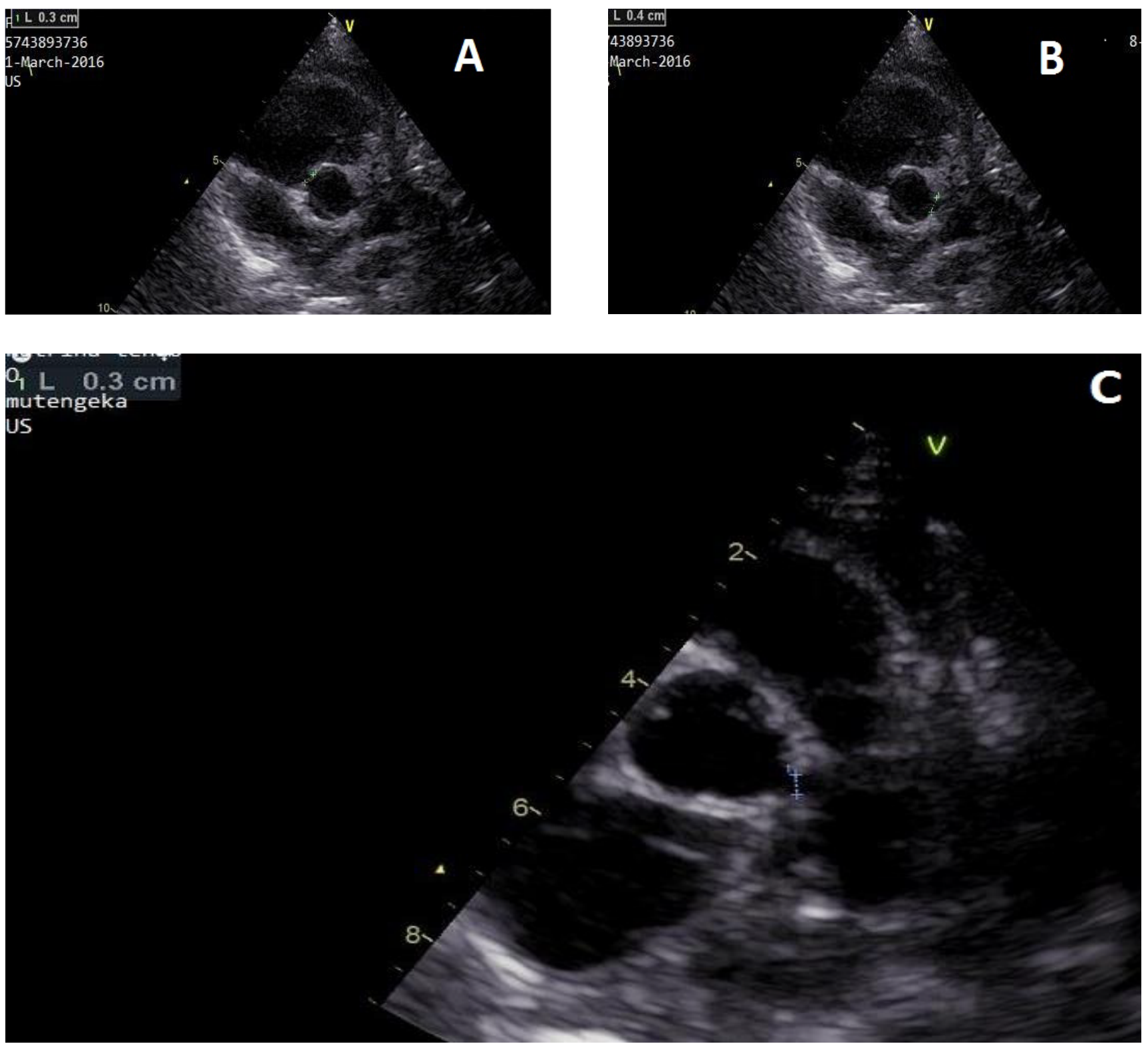

Figure: Figure showing echocardiography pictures of the dilated coronary arteries at 6 month after initiation of treatment and at 3 years of age. A) Shows dilated right coronary artery $3 \mathrm{~mm}$ in diameter; B) shows dilated left main coronary artery $4 \mathrm{~mm}$ in diameter; $\mathrm{C}$ ) shows the left main coronary artery measuring $3 \mathrm{~mm}$ in diameter at 3 years of age, right coronary artery also measured $3 \mathrm{~mm}$ in diameter (not shown in this picture).

according to American Heart Association [11] but in this infant it was started late due to delayed diagnosis and child had already developed coronary artery dilatation. Approximately $50 \%$ of coronary abnormalities regress within five years [20] and we are glad to mention that the coronary arteries for this child currently at 3 years of age are of normal $z$ scores of $<2.5$ for her body surface area.

\section{Summary}

$\mathrm{KD}$ is rare in Uganda and the rest of the African region as there is no available data. The infant in this case report presented in primary care with typical symptoms of KD but because of the rare occurrence of the disease, the diagnosis was missed by the clinicians initially. A pediatric cardiologist eventually diagnosed her with KD with coronary involvement based on symptoms, laboratory testing, and echocardiography findings. The prima- ry goal of timely treatment in KD is to prevent coronary artery disease. Despite late diagnosis appropriate treatment was commenced. First-line therapy for KD includes high dose IVIG and aspirin therapy which she received. However she had already developed coronary disease. She has made a full recovery with the resolution of coronary artery dilatation over a period of 2 years.

\section{Conclusion}

Although KD is rare in Uganda and the rest of the African region, clinicians in these parts of the world should have a high index of suspicion for early diagnosis and timely treatment of KD to reduce the risk of developing coronary artery complications.

\section{Declaration}

Authors declare no conflict of interest. 


\section{Acknowledgement}

We would like to acknowledge the patient and her family.

\section{References}

1. Brogan PA, Bose A, Burgner D, Shingadia D, Tulloh R, et al. (2002) Kawasaki disease: An evidence based approach to diagnosis, treatment, and proposals for future research. Arch Dis Child 86: 286-290.

2. Ramphul K, Mejias SG (2018) Kawasaki disease: A comprehensive review. Arch Med Sci Atheroscler Dis 3: e41-e45.

3. Harnden A, Takahashi M, Burgner D (2014) Kawasaki disease. BMJ.

4. Harnden A, Mayon-White R, Perera R, Yeates D, Goldacre M, et al. (2009) Kawasaki disease in england: Ethnicity, deprivation, and respiratory pathogens. Pediatr Infect Dis J 28: 21-24.

5. Saundankar J, Yim D, Itotoh B, Payne R, Maslin K, et al. (2014) The epidemiology and clinical features of kawasaki disease in australia. Pediatrics 133: e1009-e1014.

6. Levin M, Tizard EJ, Dillon MJ (1991) Kawasaki disease: Recent advances. Arch Dis Child 66: 1369-1374.

7. Marion de La Harpe, Stefano di Bernardo, Michaël Hofer, Nicole Sekarski (2019) Thirty years of kawasaki disease: A single-center study at the university hospital of lausanne. Front Pediatr 7: 11.

8. Dietz SM, van Stijn D, Burgner D, Levin M, Kuipers IM, et al. (2017) Dissecting kawasaki disease: A state-of-the-art review. Eur J Pediatr 176: 995-1009.

9. Nathan Jamieson, Davinder Singh-Grewal (2013) Kawasaki disease: A clinician's update. International Journal of Pediatrics.

10. Boven K, De Graeff-Meeder ER, Spliet W, Kuis W (1992) Atypical kawasaki disease: An often missed diagnosis. Eur J Pediatr 151: 577-580.
11. Newburger JW, Takahashi M, Gerber MA, Gewitz MH, Tani LY, et al. (2004) Diagnosis, treatment, and long-term management of Kawasaki disease: A statement for health professionals from the committee on rheumatic fever, endocarditis, and Kawasaki disease, council on cardiovascular disease in the young, American Heart Association. Pediatrics 114: 1708-1733.

12. McCrindle BW, Li JS, Minich LL, Colan SD, Atz AM, et al. (2007) Coronary artery involvement in children with kawasaki disease: Risk factors from analysis of serial normalized measurements. Circulation 116: 174-179.

13. de Zorzi A, Colan SD, Gauvreau K, Baker AL, Sundel RP, et al. (1998) Coronary artery dimensions may be misclassified as normal in kawasaki disease. J Pediatr 133: 254-258.

14. Newburger JW, Takahashi M, Burns JC, Beiser AS, Chung KJ, et al. (1986) The treatment of kawasaki syndrome with intravenous gamma globulin. N Engl J Med 315: 341-347.

15. Takahashi M, Mason W, Lewis AB (1987) Regression of coronary aneurysms in patients with kawasaki syndrome. Circulation 75: 387-394.

16. Research Committee on Kawasaki Disease (1984) Report of subcommittee on standardization of diagnostic criteria and reporting of coronary artery lesions in kawasaki disease. Japanese Ministry of Health and Welfare, Tokyo, Japan.

17. Kurotobi S, Nagai T, Kawakami N, Sano T (2002) Coronary diameter in normal infants, children and patients with Kawasaki disease. Pediatr Int 44: 1-4.

18. Belay ED, Maddox RA, Holman RC, Curns AT, Ballah K, et al. (2006) Kawasaki syndrome and risk factors for coronary artery abnormalities: United States, 1994-2003. Pediatr Infect Dis J 25: 245-249.

19. Honkanen VE, McCrindle BW, Laxer RM, Feldman BM, Schneider R, et al. (2003) Clinical relevance of the risk factors for coronary artery inflammation in Kawasaki disease. Pediatr Cardiol 24: 122-126.

20. Shulman ST, De Inocencio J, Hirsch R (1995) Kawasaki disease. Pediatr Clin North Am 42: 1205-1222. 